\section{Unusual 2-Stages Posterior Approach Surgical Treatment for Complete Fracture Dislocation of the Upper Thoracic Spine without Neurologic Deficit: A Case Report}

\section{Abstract}

Purpose: Traumatic posterior dislocation of the upper thoracic spine without neurological deficit has rarely been reported previously in the literature and most surgeons have less experience for surgical treatment for this kind of injury. We described a case of complete posterior dislocation of the T3 thoracic spine without neurological deficit, and its management with pedicle screw fixation and reduction through an posterior approach.

Methods: A 23-years-old man was threw out of the coach when the traffic accident happened on a highway. Clinical and imaging findings of the patient were described. Surgical treatment with posterior decompressive laminectomy, trans-pedicle screw fixation, and reduction was performed by the authors. We also reviewed the relevant literature.

Results: Complete reduction was achieved without neurological sequelae. To our knowledge, several similar cases reported in the literature were severe fracturedislocation below T4, and few case with upper thoracic spine without neurological deficit was reported previously.

Conclusions: Our experience proves that the unusual 2-stages posterior approach is a good choice for treating severe upper thoracic dislocation in acute phase. Therefore, the presented technique is an alternative approach for this rare injury.

Keywords: Upper thoracic spine; Fracture dislocation; Neurological deficit; Pedicle screw

\section{Bin Zhu ${ }^{1}$, Dasheng Tian ${ }^{1}$, Jun Qian' Lei Chen' \\ Shijia Zhang ${ }^{2}$ and Juehua Jing ${ }^{1}$}

1 Department of Orthopaedics, Second Department of Orthopaedics, Second Hospital, Affiliated to Anhui Medical University, Hefei, China

2 Department of Medicine, Rutgers-New Jersey Medical School, 185 South Orange Avenue, Newark, NJ 07103, USA

Corresponding author: Juehua Jing

\section{”zhu290488355@163.com}

Department of Orthopaedics, Second Hospital Affiliated to Anhui Medical University, Hefei, 230601, China.

\section{Tel: 8605513869506}

Citation: Zhu B, Tian D, Qian J, et al. Unusual 2-Stages Posterior Approach Surgical Treatment for Complete Fracture Dislocation of the Upper Thoracic Spine without Neurologic Deficit: A Case Report. Spine Res. 2015, 1:1.

\section{Introduction}

Thoracic fracture dislocations of the spine without neurological deficit are extremely rare injuries, and few reported cases are found in the literature [1-10]. This report presents our experience in the surgical treatment of a case of severe traumatic upper thoracic fracture-dislocation without neurological deficit in which the reduction, reconstruction, and stabilization were done in one stage via an entirely posterior approach. Other issues related to the management of this uncommon injury are discussed.

\section{Case Report}

A 23-years-old man was threw out of the coach when the traffic accident happened on a highway. He did not lose consciousness, and was taken to a nearby hospital by ambulance. Initial supine anterior-posterior chest radiograph showed that the gap between rib 3 and rib 4 became narrow and two right ribs (rib 7 and rib 8) broke (Figure 1). This finding led to a computed tomography (CT) and revealed a complete fracture dislocation of T3/T4 with total spondyloptosis of T3 vertebral body over T4 vertebral body in the sagittal (Figure 2), and magnetic resonance imaging showed that the signal of the T3-4 intervertebral disc and spinal cord seemed normal, and the high intensity signal of posterior ligamentous complex suggested the complete rupture of those ligaments (Figure 3).

Two hours later, the patient was brought to our hospital for 


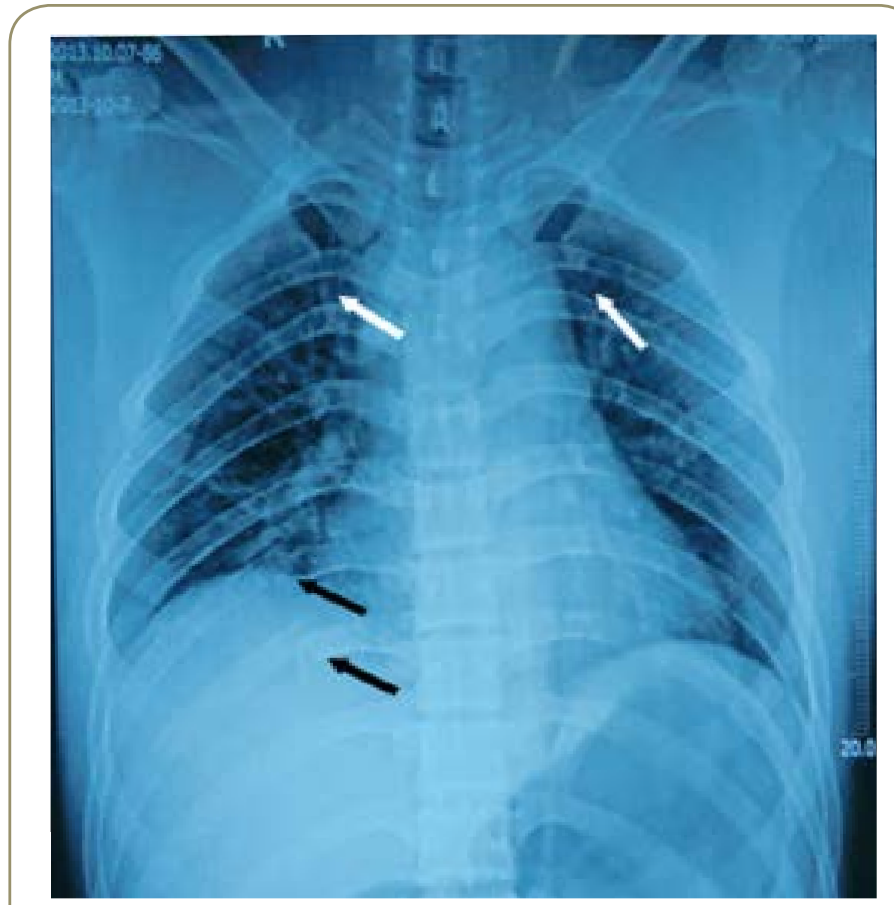

Figure 1 Preoperative anterior-posterior chest X-rays; white arrows showed that the gap between rib 3 and rib 4 became narrow and black arrows showed two right ribs(rib7 and rib 8) broke.

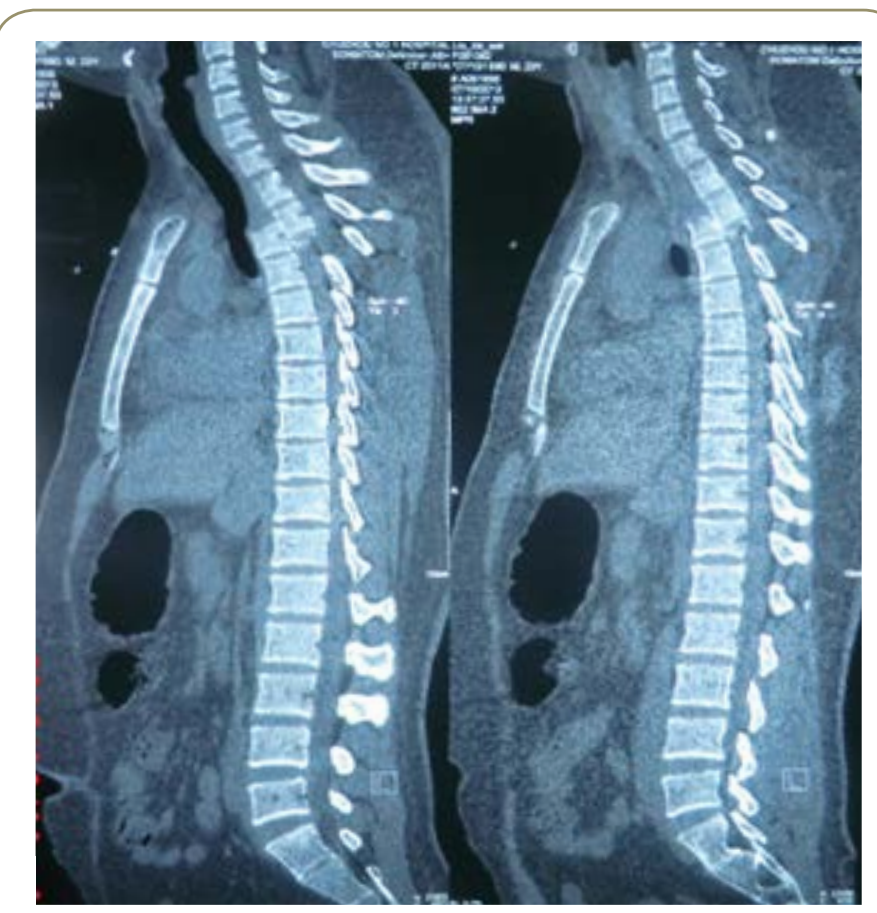

Figure 2 Sagittal reformatted computed tomography scan of the thoracic spine at presentation.

treatment. On examination, the patient's general condition was good and just complained of considerable pain in the back. Physical examination found compressive pain on chest and back, and the reflexes were normal and there was no evidence of spinal cord damage or other neural deficits.
At admission 2th day, he was undertaken posterior decompressive laminectomy, reduction, and pedicle screw fixation. Surgery was planned and executed in two steps for without spinal cord monitoring at hand. The first step in the procedure was to temporary internal with pedicle screws. In cooperation with the patient, the operation was performed under local anesthesia. The patients were positioned prone on the spinal table and secured appropriately to the bed. Care must be taken when rolling the patient. The position of the affected vertebrae (T3/ T4) was confirmed using fluoroscopy. The involved segment was exposed via midline posterior approach. The para-spinal muscles were dissected from the spinous processes to the tips of the transverse processes. Pedicle screws were placed bilaterally 2 levels above (T1/T2) and 2 levels below (T5/T6) the lesion with funnel technique under fluoroscopy guidance. Then, the screws were temporarily connected by two rods on two sides separately to avoid instability and prevent secondary neurological damage during rolling the patient and decompression, the screw caps were tightened provisionally without being torqued. Then, operative incision was closed in layers, and the patient was positioned supine.

Secondly, after general endotracheal anesthesia was administered, the patient was positioned prone again. Decompressive laminectomy of T2-T4 was performed carefully, centered at the affected level to expose the dura widely, the nerve root and the spinal cord were found to be normal except for the displacement. With the dura in direct view, the broken facet joints were removed and eliminated for they may be an obstacle to reduction. Using a rod holder to hold each rod, a distraction device straddling the rods and contacting the rod holder was applied to separate both rods longitudinally. The reduction maneuver was slight distraction to unlock the overriding segments, until the segments were reduced. The morphology of the spinal cord were observed carefully throughout each procedure while distraction was applied continuously. Then, the rods were torqued and the fusion was carried out routinely. Intra-operative a Stagnara wake-up test

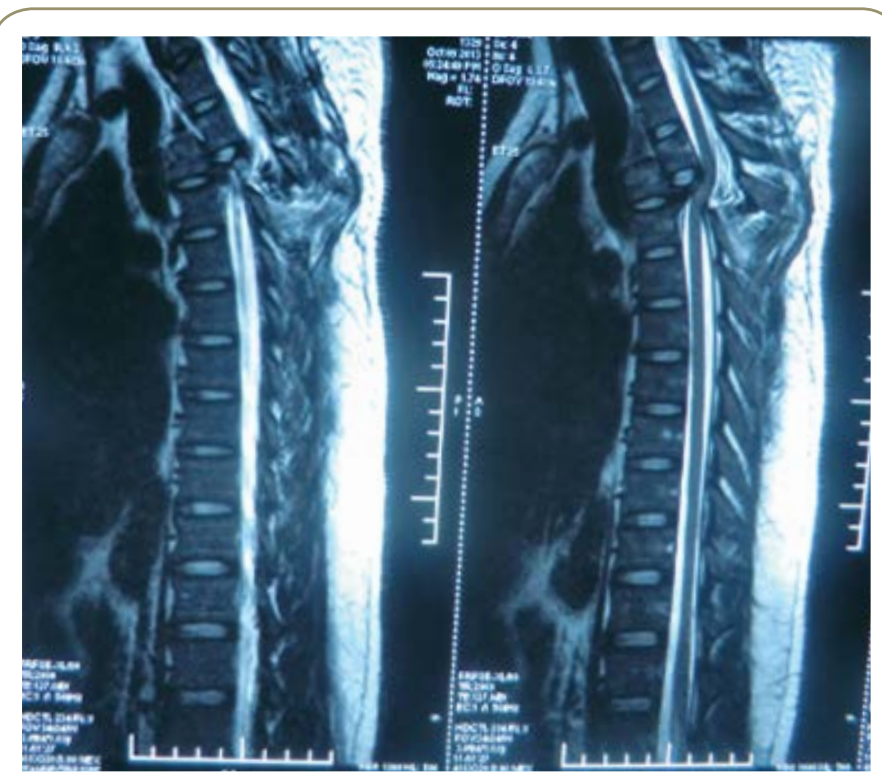

Figure 3 Preoperative sagittal MR imaging. 
was performed, the patient was evoked and his lower extremity motor functions was normal. The wound was closed in layers and a closed suction drain was left in place for 48 hours.

The operating times were 245 minutes, there was no intra- or postoperative complications. The postoperative radiographs and computed tomography showed excellent realignment of the dislocated segment (Figures 4 and 5). He could walk 6 weeks after operation. The radiographs at follow-up revealed that the fracture of the vertebra had healed and no loss of correction had been found. At latest follow-up, there was no spine-related pain. The patients were neurologically stable (ASIA Grade E).

\section{Discussion}

According to the classification by Magerl [11], the fracture-

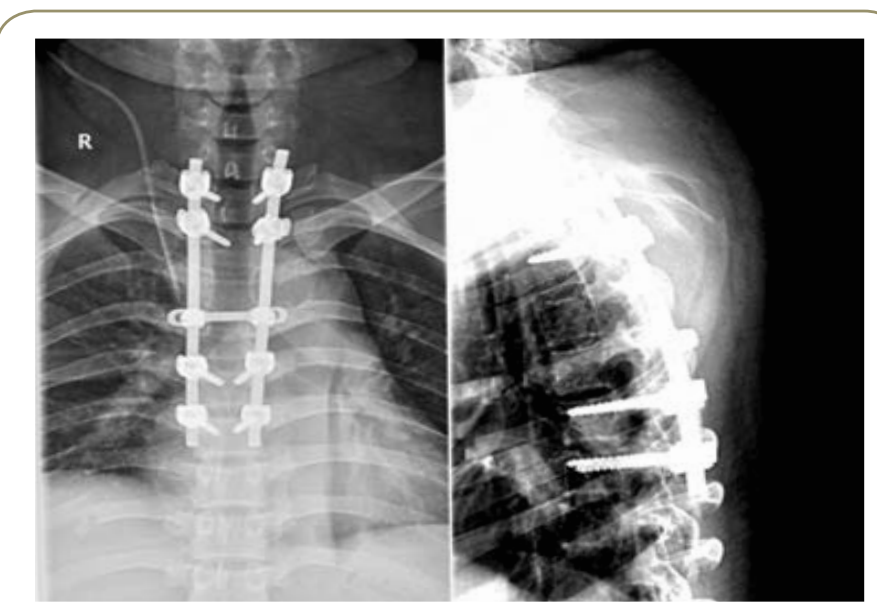

Figure 4 Postoperative radiographs after open reduction and fixation.

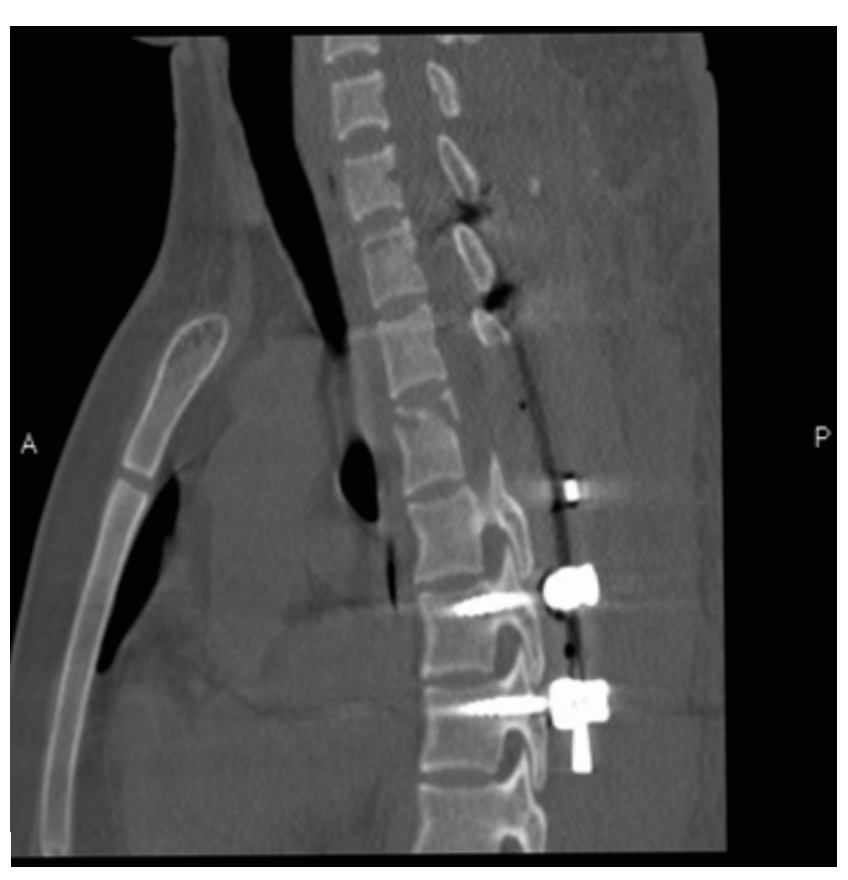

Figure 5 Postoperative sagittal reformatted computed tomography scan of the thoracic spine. dislocations described in our case can be classified as type C2.3.3, where the pure posterior dislocation following the disc lesion, together with the posterior arch fracture of T3, which remained connected to the superior segment of the spine. The intrinsic stability of thoracic provided by the anterior and posterior longitudinal ligaments, ligamentum flavum, and articulations with the ribs [12]. Therefore, a severe injury is required to bring about a fracture dislocation at this level. Most thoracic dislocations are usually associated with severe neurologic injury, or even complete paraplegia, owing to narrow spinal canal and limited vascular supply [13]. A fracture-dislocation of type B3.3 in upper thoracic spine associated without paraplegia, such as our case, is rarely reported in the literature. In 2005, F. De lure [14] described a case of the same type complete dislocation of T1-T2 without neurological deficit.

Because of the high degree of instability and poor healing associated with disco-ligamentous injuries, urgent or early surgery with decompression, reduction, and stabilization with spine fusion is the best policy in management of complete fracturedislocation of upper thoracic spine [15]. The aim of the early surgery is to restore spinal alignment and stability, to prevent further displacement and secondary neurological damage, so as to achieve maximum preservation of spinal cord function. Current recommendations for treating fracture-dislocations of the thoracic spine are operative reduction and internal fixation through a posterior approach $[16,17]$. The posterior approach is preferred for the stabilization of dislocation instability due to the ease of dissection, the relatively low incidence of complications, the high success rate of fusion, and familiarity to most surgeons [18-20]. Additionally, posterior approach can achieve a wide view of the neural canal during reduction manoeuvres.

In our case, vertebral overriding existed between the T3 and T4 vertebral. Good and safely reduction relied on effective distraction of injury space by pedicle screw and intra-operative traction. But, this reduction technique actually is very challenging and highly technically demanding. First, pedicle screw insertion in the upper and middle thoracic spine is challenging due to the specific osseous morphology and spondyloptosis. Second, it is very difficult to determine how much force applied is appropriate and over distraction has a great potential to injure the spinal cord. Third, The traction procedure must be done under repeated fluoroscopic control and continuous spinal cord monitoring which are not always available at hand. After pedicle screws were placed and superior articular facets were removed, we carried out the reduction in three phases. Temporary internal phase: two temporary distraction rods spanning the lesions with fixation points at the cephalad and caudad aspects of the lesions. We used two points of fixation at the cephalad end and two at the caudad end to achieve adequate fixation. Distraction phase: traction applied in slight extension to keep T3 vertebral apposed to T4 vertebral. T3 vertebral slowly slipped back over the T4 vertebral but was displaced far backward in sagittal view. Realignment phase: By combined traction and leverage, allowing T3 vertebral to come closer to T4 vertebral, we achieved a good reduction and realignment of the spine, as well as the complete circumferential release of the dura. 
We consider this posterior temporary internal and distraction procedure to be an excellent strategies for preserving neurological function and restoring spinal alignment in the acute phase. We recommend this technique when the spinal fracture-dislocations spine is flexible. It has been shown that most of these unstable injuries can be managed using these techniques without the need for additional combined or staged anterior spinal surgery. However, Wang and Zhu [21] pointed that deformity correction was completely achieved in all the fracture-dislocations that were subjected to surgery within 3 weeks and was partly achieved in those subjected to surgery after 3 weeks. In the subacute phase, the injury is more rigid because of some degree of fracture and tissues healing. Therefore, sometimes the posterolateral or combined approaches are a reasonable and effective option.

\section{Conclusion}

Our case provides us an experience in management of patients with complete fracture dislocation of upper thoracic spine without paraplegia. The results indicate that posterior temporary internal and distraction is a viable treatment for complete fracture dislocation of the upper thoracic spine without neurological deficit in acute phase. Clinical physicians should be more careful in patients with a diagnosis of spinal trauma without paraplegia because any maneuver of the spine may result in more a dangerous condition. Early surgical intervention is the best policy in management of this unstable spine. 


\section{References}

1 Gertzbein S, Offiersky C (1979) Complete fracture-dislocation of the thoracic spine without spinal cord injury. A case report. J Bone Joint Surg 61: 449-451.

2 Weber S, Sutherland G (1986) An unusual rotational fracturedislocation of the thoracic spine without neurologic sequelae internally fixed with a combined anterior and posterior approach. J Trauma 26: 474-479.

3 Sasson A, Mozes G (1987) Complete fracture-dislocation of the thoracic spine without deficit. A case report. Spine 12: 67-70.

4 Miyasaka Y, Satomi K, Sugihara S (1993) Posterior fracture dislocation of the thoracic spine associated without neurologic deficit. A case report and short literature review. Spine.18: 2351-2354.

5 De Lucas JC, Alvarez L, Abril JC, Calvo E (1994) Fracture-dislocation of the thoracic spine without neurological lesion. Injury 25: 105-107.

6 Korovessis P, Sidiropoulos P, Dimas A (1994) Complete fracture dislocation of the thoracic spine associated without neurologic deficit: case report. J Trauma 36: 122-124.

7 Liljenqvist U, Halm H, Castro W, Mommsen U (1995) Thoracic fracture-dislocation without spinal cord injury: a case report and literature review. Eur Spine J 4: 252-256.

8 Shapiro S, Abel T, Rodgers R (2002) Traumatic thoracic spinal fracture dislocation with minimal or no cord injury. Report of four cases and review of the literature. J Neurosurg 96: 333-337.

9 Simpson AH, Williamson DM, Golding SJ (1990) Thoracic spine translocation without cord injury. J Bone Joint Surg 72: 80-83.

10 Uriarte E, Elguezabal B, Tovio R (1987) Fracture-dislocation of the thoracic spine without neurologic lesion. Clin Orthop 217: 261-262.
11 Magerl F, Aebi M, Gertzbein SD, Harms J, Nazarian S (1994) A comprehensive classification of thoracic and lumbar injuries Eur Spine J 3: 184-201.

12 Denis F. (1983) The three column spine and its significance in the classification of acute thoracolumbar spinal injuries Spine 8: 817-883.

13 Roaf R (1960) A study of the mechanics of spinal injuries. J Bone Joint Surg 42: 810-823

14 De lure M, Fravisini S, Boriani (2005) Case report of complete dislocation of T1-T2 without neurological deficit and review of the literature.Injury Extra 36: 503-507.

15 Alobaid A, Arlet V, Ouellet J (2006) Surgical technique. Technical notes on reduction of thoracic spine fracture dislocation.Can J Surg 49: 131-134.

16 Yue J, Sossan A, Selgrath C (2002) The treatment of unstable thoracic spine fractures with transpedicular screw instrumentation: a 3-year consecutive series .Spine 27: 2782-2787.

17 McCullen G, Vaccaro A, Garfin S (1998) Thoracic and lumbar trauma. Orthop Clin North Am29: 813-828.

18 Ohtsuka K, Terayama K, Tsuchiya T, Wada K, Furukawa K et al. (1983) A surgical procedure of the anterior decompression of the thoracic spinal cord through the posterior approach. Orthop Surg Traumatol 26: 1083-1090.

19 Alobaid A, Arlet V, Ouellet J (2006) Surgical technique. Technical notes on reduction of thoracic spine fracture dislocation. Can J Surg 49: 131-134.

20 Sapkas GS, Papagelopoulos PJ, Papadakis SA (2003) Thoracic spinal injuries, operative treatments and neurologic outcomes. Am J Orthop 32: $85-88$.

21 Wang F, Zhu Y (2013) Treatment of complete fracture-dislocation of thoracolumbar spine. J Spinal Disord Tech 26: 421-426. 